\title{
Narrativa
}

narrativa

\section{Mia LECOMTE, Di un poetico altrove: poesia transnazionale italofona (1960-2016)}

\section{Gioia Panzarella}

\section{OpenEdition}

\section{Journals}

\section{Edizione digitale}

URL: https://journals.openedition.org/narrativa/566

DOI: $10.4000 /$ narrativa.566

ISSN: 2804-1224

\section{Editore}

Presses universitaires de Paris Nanterre

\section{Edizione cartacea}

Data di pubblicazione: 1 décembre 2018

Paginazione: 180-181

ISBN: 978-2-84016-325-1

ISSN: $1166-3243$

\section{Notizia bibliografica digitale}

Gioia Panzarella, «Mia LECOMTE, Di un poetico altrove: poesia transnazionale italofona (1960-2016)», Narrativa [Online], 40 | 2018, online dal 01 novembre 2021, consultato il 08 décembre 2021. URL: http://journals.openedition.org/narrativa/566 ; DOI: https://doi.org/10.4000/narrativa.566

Questo documento è stato generato automaticamente il 8 décembre 2021.

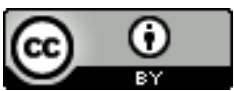

Narrativa est mise à disposition selon les termes de la Licence Creative Commons Attribution 4.0 International. 


\title{
Mia LECOMTE, Di un poetico altrove: poesia transnazionale italofona (1960-2016)
}

\author{
Gioia Panzarella
}

\section{NOTIZIA}

Mia LECOMTE, Di un poetico altrove: poesia transnazionale italofona (1960-2016), Firenze, Franco Cesati, 2018, 335 p.

1 Di un poetico altrove è una fondamentale introduzione alla poesia transnazionale italofona, una fetta di letteratura contemporanea caratterizzata dalla "vastità e fluidità delle contaminazioni" (p. 13) tra lingue, culture e forme estetiche. Lecomte passa in rassegna poeti e poetesse "che per ragioni storico-biografiche sono costretti a migrare, a porsi in transito con le proprie possibilità-impossibilità di comunicazione" (p. 16) e che si esprimono in italiano. Mentre per i testi in prosa esiste ormai un dibattito critico - che Lecomte passa in rassegna nel primo capitolo -, mancava uno studio di tale ampiezza dedicato alla produzione poetica transnazionale in lingua italiana. Già l'indice e la struttura dello studio mostrano come Lecomte si distacchi dalla narrazione ormai classica che vede i primi anni novanta come un momento di svolta in cui iniziano ad apparire i primi autori transnazionali. La sua disamina dei testi poetici transnazionali inizia infatti dagli anni sessanta e arriva ai nostri giorni. Lecomte si concentra sull'analisi di una selezione di singoli poeti e poetesse - tra cui, in ordine di presentazione, Murilo Mendes, Edith Bruck, Toni Maraini, Arnold de Vos, Egidio Molinas Leiva, Heleno Oliveira, Ubax Cristina Ali Farah, Gëzim Hajdari, Barbara Serdakowski, Božidar Stanišić, Mihai Mircea Butcovan, José Carbonero, Candelaria Romero, Julio Monteiro Martins e la Compagnia delle poete - pur tracciando una panoramica di ampio respiro grazie a un costante rimando di note e confronti con altri autori. 
Lecomte esplora le influenze e i percorsi che hanno portato ogni poeta e poetessa in Italia e alla scrittura in italiano, le "sinergie creative" tra scrittori italiani, scrittori non italiani in Italia, e i contatti con letterature in altre lingue, fino ad arrivare all'analisi della loro poetica e attraverso una selezione di versi. Lo studio di Lecomte è in linea con un approccio critico che mette in discussione la "rigidità dei canoni delle letterature nazionali, [...] quello che ancora possono significare in un contesto di transiti culturali e linguistici sempre crescenti" (p. 205). La produzione dei poeti transnazionali italofoni è quindi caratterizzata dalla non-appartenenza e per questo motivo, sottolinea Lecomte, resta in gran parte sconosciuta.

Tra le righe di Di un poetico altrove traspare la lunga esperienza di Lecomte di critica e curatrice di antologie di poesia transnazionale. Tale esperienza emerge sia dall'ampio ventaglio di testi critici di riferimento, sia dall'accesso di Lecomte a una memoria di occasioni di confronto nell'ambito di eventi e iniziative culturali, che la studiosa raccoglie in un'Appendice. Questo studio permette, inoltre, di entrare nel mondo della poesia transnazionale italofona a partire da materiale inedito, come interviste e scambi di e-mail. La ricchezza di Di un poetico altrove nasce proprio dalla prossimità di Lecomte alla produzione che presenta. È infatti significativo che l'ultimo caso analizzato, a conclusione di questo percorso, sia quello delle Compagnia delle poete, un progetto culturale e creativo promosso da Lecomte che "risponde [...] in prima istanza all'esigenza di trovare un nuovo canale espressivo per dare voce all'italofonia poetica, di individuare una nuova modalità di dialogo con la poesia 'italiana' e il suo ipotetico pubblico" (p. 239). 